\title{
Vibrio cholerae and Aeromonas: do they share a mutual host?
}

\author{
Yigal Senderovich $^{1}$, Yana Gershtein ${ }^{1}$, Etti Halewa ${ }^{2}$ and Malka Halpern ${ }^{1,2}$ \\ ${ }^{1}$ Department of Evolutionary and Environmental Biology, Faculty of Science and Science Education, \\ University of Haifa, Haifa, Israel and ${ }^{2}$ Department of Biology Education, Faculty of Science and Science \\ Education, University of Haifa, Oranim, Tivon, Israel
}

\begin{abstract}
Species of the genus Aeromonas are native inhabitants of aquatic environments and have recently been considered as an emergent human pathogen. It is estimated that aeromonads cause up to $13 \%$ of reported gastroenteritis cases in the United States. Although the autochthonous existence of Aeromonas in the aquatic environment has been established, its natural reservoir is as yet unknown. Chironomids are closely related to mosquitoes except they do not bite and they are the most widely distributed insects in freshwater. They infest drinking water systems in Israel and all over the world. Vibrio cholerae inhabit chironomids and are able to degrade their egg masses. The degradation of the egg masses is followed by failure of the eggs to hatch. In the current study, egg masses from a waste stabilization pond and a river in northern Israel were collected and cultured during a fivemonth period. Bacterial colonies were randomly chosen and checked for their egg mass degradation abilities. In addition to $\mathrm{V}$. cholerae, most of the other isolates that had the ability to degrade the egg masses were identified as Aeromonas species, thus, demonstrating that Aeromonas species are natural inhabitants of chironomid egg masses. The following virulence-associated genes were detected in Aeromonas species that were isolated from chironomid egg masses: alt (78\%); ahpB (76\%); act/aerA/hlyA (65\%); fla (59\%); pla/lipH3/apl-1/lip (43\%); and ast (2\%). These findings indicate that the Aeromonas species inhabiting chironomid egg masses pose a potential health risk. Understanding the natural reservoir of Aeromonas will help to develop methods to monitor and control the bacteria in fresh and drinking water reservoirs and to better understand the relationships between chironomids, V. cholerae and Aeromonas populations.
\end{abstract}

The ISME Journal (2008) 2, 276-283; doi:10.1038/ismej.2007.114; published online 7 February 2008

Subject Category: microbe-microbe and microbe-host interactions

Keywords: Aeromonas; chironomid; egg mass; host; reservoir; Vibrio cholerae

\section{Introduction}

The genus Aeromonas are waterborne Gram-negative bacteria that are ubiquitous in water, including groundwater and chlorinated drinking water (LeChevallier et al., 1982; Kühn et al., 1997a, b; Gavriel et al., 1998). Worldwide studies have demonstrated that Aeromonas spp are universally distributed and widely isolated from clinical and environmental samples (Nakano et al., 1990; Huys et al., 1996). Aeromonas comprise important human pathogens, including A.hydrophila, A. veronii and A. caviae (also known as $A$. punctata), which cause gastroenteritis, primary and secondary septicemia, and wound infections. Aeromonas strains isolated from water have been shown to possess virulence traits, such as adhesins, hemolysins and cytotonic

Correspondence: M Halpern, Department of Biology Education, Faculty of Science and Science Education, University of Haifa, Oranim, Tivon 36006, Israel.

E-mail: mhalpern@research.haifa.ac.il

Received 18 September 2007; revised 22 November 2007; accepted 4 December 2007; published online 7 February 2008 enterotoxins, presumably involved with human pathogenicity (Handfield et al., 1996; Albert et al., 2000; Schubert, 2000). The prevalence of Aeromonas species in the aquatic environment has been recognized as a potential health risk, and some countries have adopted aeromonad counts as an additional indicator of water quality. For example, $A$. hydrophila has been placed on the US Environmental Protection Agency's Contaminant Candidate List of emerging pathogens in drinking water (Borchardt et al., 2003). Although it is clear that members of Aeromonas are primarily aquatic organisms, their natural host in the aquatic habitat is as yet unknown.

Among aquatic invertebrates, the nonbiting midges (Chironomidae, Diptera) are globally distributed, and they are the most abundant group of insects found in freshwater ecosystems (Armitage et al., 1995). They undergo four life stages, three of which are aquatic-egg, larva and pupa-and the last of which is the adult stage, when the insect emerges from the water into the air. After mating, the adult female lays a gelatin egg mass on the water's edge, with each mass containing hundreds of 
embedded eggs. Egg masses of Chironomus sp were found to harbor Vibrio cholerae, and they provide a natural reservoir for this bacterium (Broza and Halpern, 2001; Halpern et al., 2004, 2006). It was found that chironomid egg masses were destroyed in the presence of $V$. cholerae, and degradation of the egg masses was followed by failure of the eggs to hatch (Broza and Halpern, 2001). Hemagglutinin/ protease, an extracellular enzyme of $V$. cholerae, was purified from $V$. cholerae $\mathrm{O9}$ and from the pathogenic $\mathrm{O} 1$ and $\mathrm{O} 139$ serogroups as the enzyme responsible for the degradation of chironomid egg masses (Halpern et al., 2003). Furthermore, it was found that all $V$. cholerae strains, which were isolated from chironomid egg masses were able to degrade chironomid egg masses (Halpern et al., $2003,2006,2007)$. An interaction of host-pathogen population dynamics was found between the chironomid population and $V$. cholerae in their egg masses, and a peak in the chironomid population was followed by a peak in the $V$. cholerae population (Halpern et al., 2006).

The primary objective of this study was to explore bacteria other than $V$. cholerae that inhabit chironomid egg masses and are able to degrade the egg masses. Most of the isolates that have shown the ability to degrade the egg masses have been identified as Aeromonas species, demonstrating that Aeromonas species are natural inhabitants of chironomid egg masses. This study will allow us to understand the relationships between chironomids, $V$. cholerae and Aeromonas populations better. Moreover, since the prevalence of Aeromonas species in the aquatic environment has been recognized as a potential health risk, understanding the natural reservoir of Aeromonas will help in developing methods to monitor and control the bacteria in fresh and drinking water reservoirs.

\section{Materials and methods}

Sampling sites and environmental parameters

The study was performed in northern Israel near Haifa at two sampling sites: the Tivon waste stabilization pond (WSP; longitude 699, latitude 618 ) and the Kishon River (near Kiryat Haroshet; longitude 697, latitude 620). The sites were within a radius of 2 miles. Water temperature, $\mathrm{pH}$ (Scan Model2, Rajah Crescent, Singapore) and conductivity (El-Hamma Model TH-250, Mevo Hamma, Israel) were routinely monitored. Fluctuations of 11.3 and $8.1^{\circ} \mathrm{C}$ in water temperature were measured across the sampling months in the Tivon WSP and the Kishon River, respectively. Maximal temperature $\left(33.5^{\circ} \mathrm{C}\right)$ was measured on June 23 in the Tivon WSP. The water temperatures were always higher in the Tivon WSP than the water temperatures in the Kishon River. A basic pH was measured in both the Tivon WSP $(7.8 \pm 0.36)$ and the Kishon River $(8.7 \pm 0.24)$. The conductivity that was measured in the Tivon WSP was between 1.5 and $1.8 \mathrm{mmoh} \mathrm{cm}^{-1}$ and it was three times lower than that of Kishon River (between 4.0 and $5.9 \mathrm{mmoh} \mathrm{cm}^{-1}$ ).

\section{Egg mass sampling}

Egg masses were sampled twice each month between May and September 2005. Styrofoam boards $(25 \times 25 \mathrm{~cm})$ were used as artificial oviposition sites for adult females. Egg masses along the side of the board were collected $24 \mathrm{~h}$ after introducing the board into the water habitat.

\section{Isolation of $\mathrm{V}$. cholerae and chironomid egg mass-degrading bacteria}

Chironomid egg masses from both sampling sites were brought to the laboratory immediately after collection. Three egg masses from each sampling site were chosen randomly for isolation of the bacteria. Each freshly collected egg mass was treated separately to remove all the bacteria that were not firmly attached to the egg mass. After washing five times and vortexing for $1 \mathrm{~min}$ in sterile saline water, each egg mass was crushed in $1 \mathrm{ml}$ saline, using a sterile glass homogenizer. The homogenate was diluted, and $0.1 \mathrm{ml}$ from each dilution was then spread onto thiosulfate-citrate-bile salts (TCBS; Becton, Dickson and Company, Sparks, MD, USA) for isolation of $V$. cholerae strains. To screen for other bacterial species with the potential ability of degrading the egg masses, samples from each dilution were also spread onto the following media: (i) cetrimide agar base (Himedia, Mumbai, Maharashtra, India); (ii) modified cetrimide agar base (no glycerol; gelatin-15 $\mathrm{gl}^{-1}$ ); (iii) modified minimal broth Davis without dextrose (Becton, Dickson and Company)_gelatin and yeast extract were added, $15 \mathrm{gl}^{-1}$ and $0.5 \mathrm{gl}^{-1}$, respectively; and (iv) LB agar (Luria) (Himedia). Yellow colonies from the TCBS medium that were suspected to be $V$. cholerae were subcultured on LB agar and further examined as described below. Individual colonies from all the other four media were randomly picked and streaked onto LB agar to obtain single colonies. All isolated colonies were subcultured at least four times before further examination. Bacterial isolates were kept in LB with $30 \%$ glycerol $\left(-80^{\circ} \mathrm{C}\right)$.

Identification of $\mathrm{V}$. cholerae

Yellow colonies from the TCBS medium that were suspected to be $V$. cholerae were subcultured on LB agar and then tested for oxidase (1\% tetramethyl$p$-phenylenediamine; Sigma, Saint Louis, MO, USA), as well as for the string test $(0.5 \%$ sodium deoxycholate; Sigma). The identity of the isolates with positive results in the above tests was further verified by using the multiplex PCR assay in accordance with Nandi et al. (2000). The presence of toxin genes (in addition to $\operatorname{ctx} A$, as described in 
Nandi et al., 2000) stn/sto, hlyA, hapA and ompU were determined in all $V$. cholerae strains. The primers used and the PCR procedure is described in Halpern et al. (2006) (Table 1).

\section{Egg mass-degradation activity}

All the isolates (ca. 1100) were tested for their ability to degrade chironomid egg masses. Bacterial isolates were grown in $5 \mathrm{ml} \mathrm{LB}$ medium in a shaker incubator at $30^{\circ} \mathrm{C}$ (or $37^{\circ} \mathrm{C}$ for $V$. cholerae isolates) for $48 \mathrm{~h}$. Culture supernatants were obtained by centrifugation (12000 r.p.m. for $7 \mathrm{~min}$ ) and then were filtered through a $0.45-\mu \mathrm{m}$ pore size filter (Corning; Corning, New York, NY, USA), which resulted in a clear supernatant. Degradation activity was determined by using freshly collected or ethanol (70\%)-rinsed chironomid egg masses. Degradation assay was performed by incubating one egg mass in $1.0 \mathrm{ml}$ clear supernatant at $30^{\circ} \mathrm{C}$ for up to $72 \mathrm{~h}$. Each test was carried out at least three times. A reaction was considered positive when the egg mass structure disintegrated and a large excess of free eggs was found separated from the egg mass, as compared with the control LB medium (see Figure 1). Positive results were obtained between 10 and $40 \mathrm{~h}$.

Identification of egg mass-degrading bacteria Universal bacterial primers: 8F (5'-CACGGATCCAGAC TTTGAT(C/T)(A/C)TGGCTCAG-3') and 1512R (5'-GTG AAGCTTACGG(C/T)TAGCTTGTTACGACTT-3'), based on Escherichia coli positions, were used to amplify internal fragments of $16 \mathrm{~S}$ rRNA gene (Felske et al., 1997). Eight microliters of a bacterial suspension was transferred to a sterile thin-walled PCR tube. One microliter of each primer $\left(20\right.$ pmole $\left.\mu \mathrm{l}^{-1}\right)$ and $10 \mu \mathrm{l}$ of PCR master mixture (ReddyMix, ABgene,
Epsom, UK) were added to the tube to a final reaction volume of $20 \mu \mathrm{l}$. Initial DNA denaturation was performed at $94{ }^{\circ} \mathrm{C}$ for $4 \mathrm{~min}$ followed by 33 cycles of denaturation at $94{ }^{\circ} \mathrm{C}$ for $30 \mathrm{~s}$, annealing at $55{ }^{\circ} \mathrm{C}$ for $50 \mathrm{~s}$, and elongation at $72^{\circ} \mathrm{C}$ for $2 \mathrm{~min}$, and then a final elongation step at $72{ }^{\circ} \mathrm{C}$ for $10 \mathrm{~min}$. The amplified PCR products were purified with the AccuPrep PCR purification kit (BIONEER, Daedeok-gu, Deajeon, South Korea). Purified PCR products were sequenced at Technion Medical School (Haifa, Israel) directly by the dideoxynucleotide chain-termination method using a DNA sequencer (ABI PRISM 3100) with BigDye

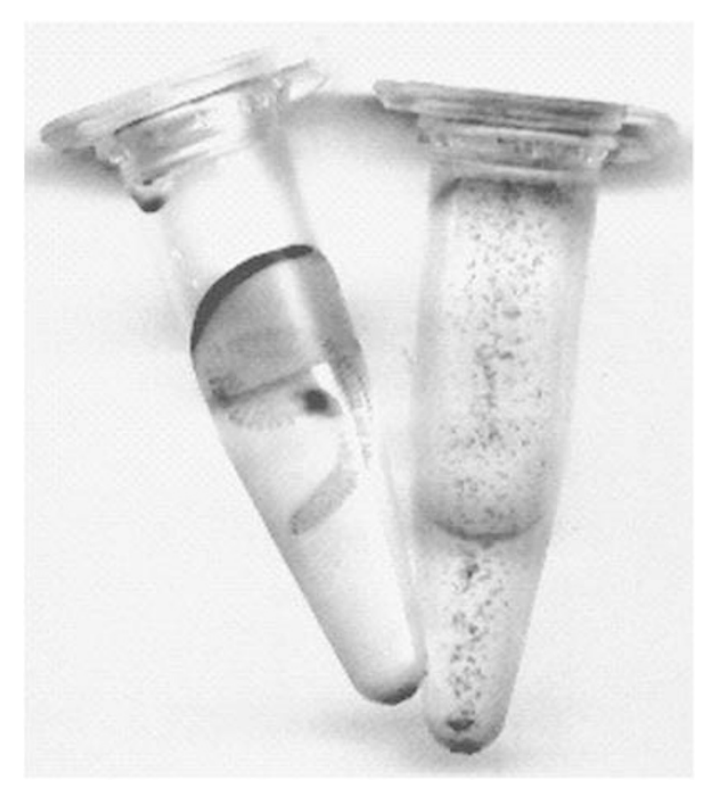

Figure 1 Chironomid egg masses before (left) and after (right) $10 \mathrm{~h}$ incubation with the supernatant of a 48-h culture of Vibrio cholerae. The eggs were freed from the degraded gelatinous matrix.

Table 1 The presence of $V$. cholerae or Aeromonas species among the bacteria that were cultured from chironomid egg masses on all the sampling dates

\begin{tabular}{|c|c|c|c|c|}
\hline \multirow[t]{2}{*}{ Sampling (month, 2005) } & \multicolumn{2}{|c|}{ Tivon WSP } & \multicolumn{2}{|c|}{ Kishon River } \\
\hline & V. cholerae & Aeromonas & V. cholerae & Aeromonas \\
\hline 1 (4 May) & + & $t^{\mathrm{a}}$ & ND & ND \\
\hline 2 (18 May) & + & + & ND & ND \\
\hline 3 (8 June) & + & + & + & + \\
\hline 4 (23 June) & - & - & + & - \\
\hline 5 (7 July) & + & $t^{\mathrm{a}}$ & + & - \\
\hline 6 (21 July) & - & $t^{\mathrm{a}}$ & - & - \\
\hline 7 (1 August) & + & + & ND & ND \\
\hline 8 (10 August) & + & + & + & - \\
\hline 9 (24 August) & + & + & + & - \\
\hline 10 (7 September) & + & + & + & + \\
\hline 11 (19 September) & + & + & - & + \\
\hline
\end{tabular}

Abbreviation: ND, no egg masses were sampled on this sampling date.

Aeromonas was also detected in DNA extracted from egg masses that were sampled from the Tivon WSP by aero-specific primers for Aeromonas. DNA was not extracted from egg masses that were sampled from the Kishon River.

${ }^{a}$ Aeromonas was not cultured but was detected in the egg mass by using aero primers for the detection of Aeromonas sp. 
terminator reagents (Applied Biosystems, Foster City, CA, USA) according to the manufacturer's instructions. The sequencing was performed by using $8 \mathrm{~F}$ primer and for several isolates-the reverse 1512 primer was used as well. This resulted in data for approximately 750 or $1504 \mathrm{bp}$, respectively. Newly determined sequences were compared to those available in the GenBank (http://www. ncbi.nlm.nih.gov) databases, using the standard nucleotide-nucleotide BLAST program (BLASTN; http://www.ncbi.nlm.nih.gov), to ascertain their closest relatives. These sequence data have been submitted to the GenBank database under accession numbers EF634206-EF634234 (see also Supplementary Table S1). A phylogenetic tree was generated by using the neighbor-joining method with NJPlot (MEGA 3), based on alignments from CLUSTAL W. The bootstrap values obtained were from 1000 iterations.

\section{Identification of isolates using specific primers for Aeromonas}

Since most isolates were identified as Aeromonas species while using 16S rRNA gene sequencing, the identification of the remaining isolates was done by using specific primers for the genus Aeromonas and for four species in this genus: $A$. hydrophila, A. punctata, $A$. veronii and $A$. schubertii. All nucleotide primers were targeted to the 16S-23S intergenic spacers, in accordance with Kong et al. (1999). To confirm amplicon production, the mixture was analyzed by electrophoresis on $1.5 \%$ agarose gel, followed by staining with ethidium bromide and visualization under ultraviolet light.

Direct identification of Aeromonas spp presence in egg masses using PCR

DNA was extracted from individual egg masses that were sampled in Tivon WSP as follows: Each egg mass was first incubated with proteinase $\mathrm{K}$ and then DNA was extracted by using Invisorb Spin Plant Mini Kit (Invitec, Berlin, Germany), according to the manufacturer's instructions. The presence of Aeromonas was detected in the extracted DNA by using aero-F and aero-R primers (Kong et al., 1999), specific for the detection of members of the Aeromonas genus.

\section{Genotypes associated with virulence in isolates of} Aeromonas species

The presence of the following genes coding for the virulence factors, was determined in all Aeromonas isolates: act/aerA/hlyA by using one set of primers AHCF1/AHCR1; alt and ast genes for cytotonic enterotozins; ahyB genes for elastase; pla/lipH3/ apl-1/lip genes for phospholipase; and fla gene for flagellin (Kingombe et al., 1999; Sen and Rodgers, 2004). The presence of the genes act/aerA/hlyA and ast; fla and alt; ahyB and pla/lipH3/apl-1/lip was tested simultaneously in the same reaction mixture, according to Sen and Rodgers (2004).

\section{Results}

Chironomid egg mass-degrading bacteria

A total number of approximately 1100 bacterial colonies were isolated from chironomid egg masses between May and September 2005. All isolates were examined for their ability to degrade chironomid egg masses. The supernatants of all the isolates that were identified as $V$. cholerae (82 isolates) were able to degrade the egg masses within $10 \mathrm{~h}$ at the most (Figure 1). Only 45 out of all the rest of the isolates (1018), which were randomly picked from the different culture media and were not identified as $V$. cholerae, were able to degrade the egg masses. These degrading bacteria were identified either by the 16S rRNA gene or by using specific primers for Aeromonas species. Thirty-three isolates were identified as $A$. punctata, nine as $A$. veronii, one as $A$. hydrophila, one as Pseudomonas otitidis and one as P. pseudoalcaligenes (see Figure 2 and Supplementary Tables S1 and S2). The time required for the egg mass degradation by the 48-h supernatant ranged between 15 and $40 \mathrm{~h}$, and there was no clear association with particular bacterial species (Supplementary Tables S1 and S2). The phylogenetic relationships between the egg mass-degrading isolates and their closest relatives from public databases are shown in Figure 2.

During the 5 month period, 11 samplings were performed at the two sampling sites. Table 1 summarizes the presence of $V$. cholerae and/or Aeromonas species among the bacteria that were cultured from chironomid egg masses on all the sampling dates. DNA was extracted from egg masses that were collected on all the sampling dates from the Tivon WSP, but not from the Kishon River. The primers aero-F and aero- $\mathrm{R}$ were used to amplify a 550-bp amplicon, demonstrating the presence of Aeromonas sp in all the sampled egg masses from the Tivon WSP (except for sampling 4, see Table 1). All the egg masses were washed and vortexed several times before they were cultured or before DNA was extracted. Thus, the detected or isolated bacteria were attached to the egg masses. No enrichment procedures were used to isolate $V$. cholerae. Likewise, no enrichment or selective media were used for the isolation of Aeromonas species.

Genotype of V. cholerae isolates

None of the strains $(n=82)$ that were isolated from the egg masses and examined in this study contained the genes for the cholera toxin $(\operatorname{ct} x A)$ or for non-O1 heat-stable enterotoxin (stn/sto). The genes for El Tor-like hemolysin (hlyA) and outer 


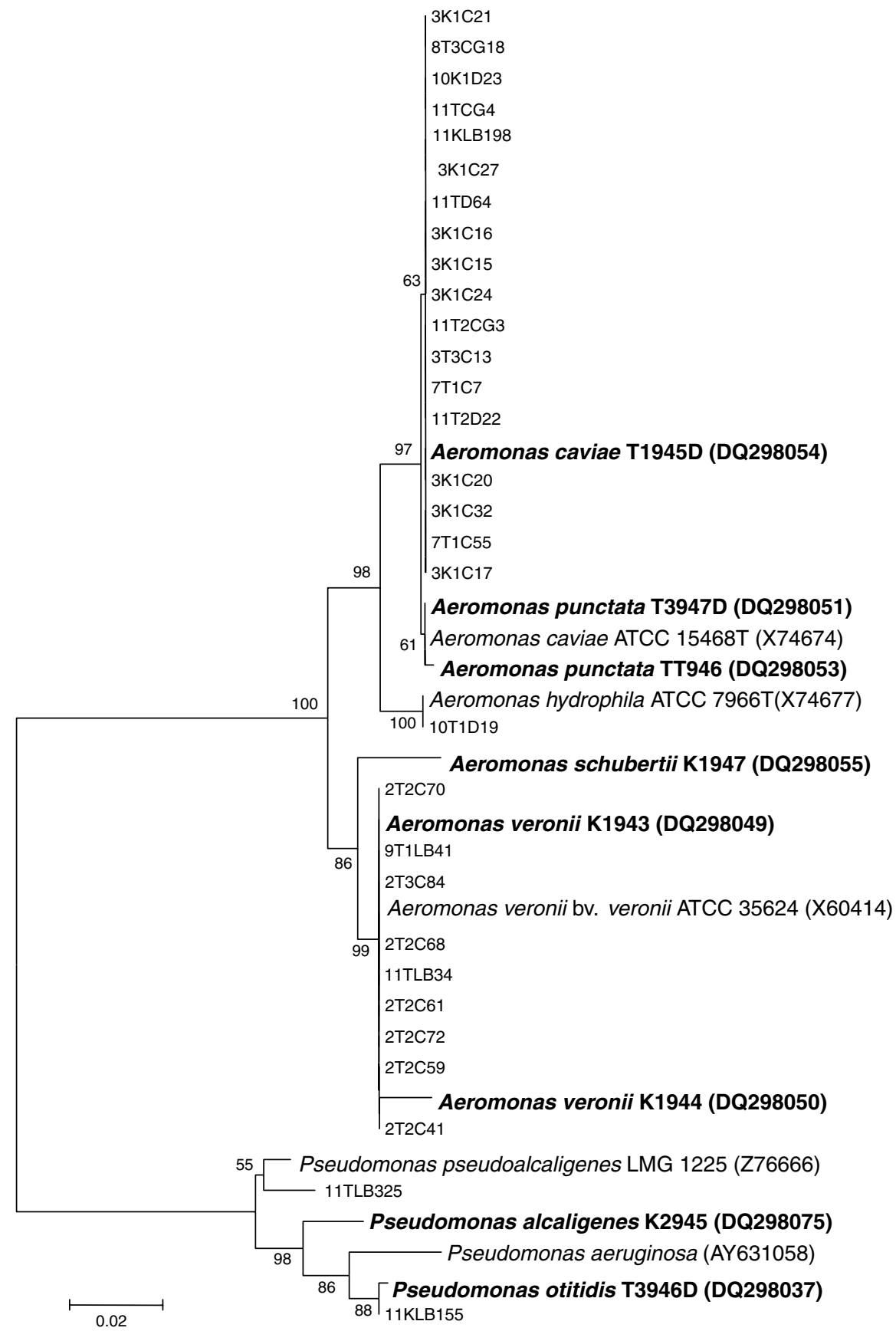

Figure 2 Phylogenetic tree of chironomid egg mass-degrading bacteria isolated from the egg masses. The tree shows the relationships based on partial sequences of the $16 \mathrm{~S}$ ribosomal RNA gene. Strains in bold were previously isolated from chironomid egg masses (Halpern et al., 2007). The accession numbers of the isolates and the time required for egg mass degradation are listed in Supplementary Table S1. The sequences alignments were performed using the CLUSTAL W program, and the tree was generated using the neighborjoining method with Kimura 2 parameter distances in MEGA 3 software. Bootstrap values (from 1000 replicates) greater than $50 \%$ are shown at the branch points. The bar indicates $2 \%$ sequence divergence.

membrane protein (ompU), which have been associated with enteropathogenicity, were found in $70 \%$ and $16 \%$ of the strains, respectively. The genotypes were found in various combinations (see Table 2). All isolates possessed the structural gene hapA, which is responsible for soluble hemagglutinin/ protease production (see Table 2). The supernatants of all the strains examined in this study demonstrated the ability to degrade chironomid egg masses.

Virulence factors in Aeromonas species

Aeromonas bacteria that were isolated from the chironomid egg masses were screened for the presence of genes for six virulence factors: act/aerA/hly $A$ genes 
Table 2 Genotypic traits of representative isolates of $V$. cholerae non-O1/non-O139 examined in this study $(n=82)$

Genotype

omp $W^{+}$hap $A^{+}$hly $A^{+}$ompU

omp $W^{+}$hap $A^{+}$hly $A^{+}$ompU $U^{-}$

omp $W^{+}$hap $A^{+}$hly $A^{-}$ompU $U^{+}$

omp $W^{+}$hap $A^{+}$hly $A^{-}$ompU

All the examined strains had the genotype of $\operatorname{ctx} A^{-}$and $\operatorname{stn} / \mathrm{sto}^{-}$.

Table 3 Genotypic traits of Aeromonas spp isolated from chironomid egg masses $(n=43)$

\begin{tabular}{|c|c|}
\hline Genotype & Presence (\%) \\
\hline pla/lipH3/apl-1/lip $p^{+}$ahpB $B^{+}$ast $^{-}$act $^{+}$fla $^{+}$alt $^{+}$ & 26 \\
\hline pla/lipH3/apl-1/lip- ahpB ${ }^{+}$ast $^{-}$act $^{+}$fla $^{+}$alt $^{+}$ & 12 \\
\hline pla/lipH3/apl-1/lip ${ }^{+}$ahpB $B^{+}$ast $^{-}$act $^{-}$fla $^{+}$alt $^{+}$ & 7 \\
\hline pla/lipH3/apl-1/lip- ahpB ${ }^{-}$ast $^{-}$act $^{+}$fla $^{+}$alt $^{+}$ & 9 \\
\hline pla/lipH3/apl-1/lip ${ }^{+}$ahpB $B^{+}$ast $^{-}$act $^{-}$fla $^{-}$alt $^{+}$ & 7 \\
\hline pla/lipH3/apl-1/lip ${ }^{+}$ahpB ${ }^{+}$ast $^{-}$act $^{-}$fla $^{+}$alt $^{-}$ & 2 \\
\hline pla/lipH3/apl-1/lip ${ }^{-} a h p B^{+}$ast $^{-}$act $^{+}$fla $^{-}$alt $^{+}$ & 2 \\
\hline pla/lipH3/apl-1/lip ${ }^{-}$ahpB ${ }^{+}$ast $^{-}$act $^{+}$fla $^{+}$alt $^{-}$ & 2 \\
\hline pla/lipH3/apl-1/lip ${ }^{+}$ahpB $B^{+}$ast $^{-}$act $^{+}$fla $^{-}$alt $^{-}$ & 2 \\
\hline pla/lipH3/apl-1/lip ${ }^{-}$ahpB $B^{+}$ast $^{-}$act $^{-}$fla $^{-}$alt $^{+}$ & 9 \\
\hline pla/lipH3/apl-1/lip $p^{-}$ahpB $B^{-}$ast act $^{-}$fla $^{-}$alt $^{+}$ & 7 \\
\hline pla/lipH3/apl-1/lip ${ }^{-}$ahpB $B^{+}$ast $^{-}$act $^{+}$fla $^{-}$alt $^{-}$ & 2 \\
\hline pla/lipH3/apl-1/lip ${ }^{-}$ahpB $B^{+}$ast $^{+}$act $^{-}$fla $^{-}$alt $^{-}$ & 2 \\
\hline pla/lipH3/apl-1/lip ${ }^{-}$ahpB $B^{+}$ast $^{-}$act $^{-}$fla $^{-}$alt $^{-}$ & 5 \\
\hline pla/lipH3/apl-1/lip ${ }^{-}$ahpB $B^{-}$ast $^{-}$act $^{+}$fla $^{-}$alt $^{-}$ & 2 \\
\hline pla/lipH3/apl-1/lip $p^{-}$ahpB $B^{-}$ast ${ }^{-}$act fla $^{-}$alt $^{-}$ & 2 \\
\hline
\end{tabular}

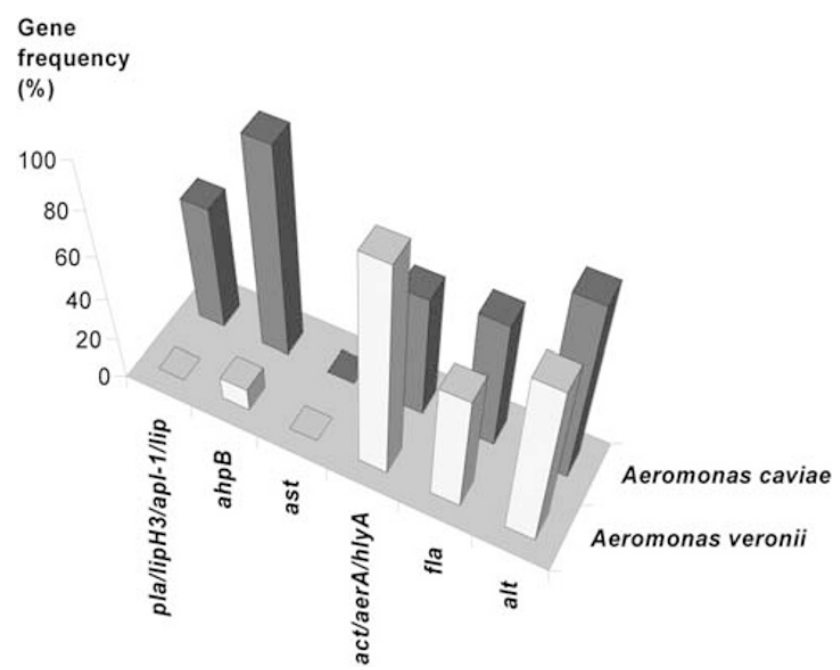

Figure 3 The frequency of the virulence genes among $A$. veronii $(n=9)$ and $A$. caviae $(n=33)$ isolated from chironomid egg masses.

for a cytotoxic enterotoxin and hemolytic activity; ast and alt genes for cytotonic enterotoxins; $a h p B$ gene for elastase; pla/lipH3/apl-1/lip genes for lipase; and fla gene for flagella. The most prevalent genes were alt and $a h p B$, which were found in $78 \%$ and $76 \%$ of the isolates, respectively (see Table 3,
Figure 3). The genes act/aerA/hlyA, fla and pla/ lipH3/apl-1/lip were also quite prevalent among the isolates $(65 \%, 59 \%$ and $43 \%$, respectively). The most infrequent gene was ast (see Table 3, Figure 3). It was found only in one isolate, which was identified as $A$. hydrophila (isolate 10T1D19) (see Figure 2 and Supplementary Table S1). The isolate 9T1LB56 was the only one that did not possess any of the six examined virulence factors. Although the virulence genotypes were found in different combinations, five of the six examined virulence factors were positive (ast was negative) in the most widespread genotype (26\% of all isolates) (see Table 3 ).

\section{Discussion}

Chironomid egg masses are known to be a natural reservoir of V. cholerae (Broza and Halpern, 2001; Halpern et al., 2004, 2006, 2007). While exploring for bacteria other than $V$. cholerae that can degrade the egg masses, we demonstrated that chironomid egg masses harbor Aeromonas species along with $V$. cholerae. Aeromonas species were isolated from the egg masses throughout most of the samplings and were further shown to be inhabitants of the egg masses by using specific primers for Aeromonas sp (Table 1).

In a recently published study, Aeromonas species were isolated from chironomid egg masses when the bacterial diversity of the egg masses was studied (Halpern et al., 2007). Both in Halpern et al. (2007) and in the current study, egg masses were thoroughly washed to obtain only bacteria that were attached, probably as a biofilm, to the egg mass and no selective or enrichment methods were used for the isolation of Aeromonas species. Thus, Aeromonas species seem to be inhabitants of chironomid egg masses.

The following virulence-associated genes were detected in $V$. cholerae isolates from chironomid egg masses: hemagglutinin/protease (hapA, 100\%); hemolysin (hlyA, 70\%); and outer membrane protein (ompU, 16\%) (Table 2). Cholera toxin (ctxA) and the non-O1 heat-stable enterotoxin $(s t n / s t o)$ were not detected. A very similar pattern (hapA 100\%, hlyA $72 \%$ and ompU 28\%) was observed in a previous study examining $V$. cholerae isolates from egg masses (Halpern et al., 2006).

The following virulence-associated genes were detected in Aeromonas in the current study: 78\% (alt), 76\% (ahpB), 65\% (act/aerA/hlyA), 59\% (fla), $43 \%$ (pla/lipH3/apl-1/lip) and 2\% (ast) (see Table 3, Figure 3). These results (except for the ast frequencies) are quite similar to those obtained by Sen and Rodgers (2004): 88\% (ahyB), 88\% (lip), 59\% (fla), $43 \%$ (alt), $70 \%$ (act) and $30 \%$ (ast). The genes were found in various combinations in both studies. The results show that Aeromonas bacteria found in chironomid egg masses possess a wide variety of virulence-related genes, as was shown by Sen and 
Rodgers (2004) for bacteria that were isolated from drinking water.

Chironomid infestation in drinking water supply systems is an existing problem in Israel (Halpern et al., 1999, 2002) and in other countries in the world, for example, the United Kingdom (Williams, 1974), the United States (Bay 1993; Alexander et al., 1997), China (Sun et al., 2007) and Japan (Tanaka et al., 2004). In Japan, the source of chironomids in tap water contamination was suggested to be the larvae, which live in the water purification plant (Tanaka et al., 2004). Chironomids may disseminate pathogenic species of Aeromonas between drinking water reservoirs, as was suggested for $V$. cholerae (Broza et al., 2005). Furthermore, the gelatinous egg mass matrix may protect Aeromonas that inhabit the egg masses from the disinfecting agents, which are used in the drinking water systems.

No correlation was found between the concentration of Aeromonas spp and fecal indicator organisms (Legnani et al., 1998). However, Aeromonas species showed a seasonal distribution, as was found for chironomids (Holmes et al., 1996; Halpern et al., 2004, 2006). In surface water, Aeromonas abundance peaks in the warm summer and fall months (Burke et al., 1984; Chauret et al., 2001). The seasonal increase in Aeromonas detection in the drinking water supply was found to match the peak occurrence of clinical isolates (Burke et al., 1984).

Since the prevalence of Aeromonas species in the aquatic environment has been recognized as a potential health risk (Borchardt et al., 2003), understanding the natural reservoir of Aeromonas will help in developing methods to monitor and control the bacteria in fresh and drinking water reservoirs. More work is needed to survey Aeromonas abundance in chironomid egg masses in different freshwater habitats, including drinking water reservoirs, so as to identify the secreted factor that degrades the egg mass and to monitor the population dynamics of chironomids, Aeromonas and V. cholerae, and their dependency.

\section{References}

Albert MJ, Ansaruzzaman M, Talukder KA, Chopra AK, Kühn I, Rahman M et al. (2000). Prevalence of enterotoxin genes in Aeromonas spp. isolated from children with diarrhea, healthy controls, and the environment. J Clin Microbiol 38: 3785-3790.

Alexander MK, Merrit RW, Berg MB. (1997). New strategies for the control of the parthenogenetic chironomid (Paratanytarsus grimmii) (Diptera: Chironomidae) infesting water systems. J Am Mosq Control Assoc 13: 189-192.

Armitage P, Cranston PS, Pinder LCV. (1995). The Chironomidae: The Biology and Ecology of Non-Biting Midges. Chapman \& Hall: London, UK.

Bay EC. (1993). Chironomid (Diptera: Chironomidae) larval occurrence and transport in a municipal water system. J Am Mosq Control Assoc 9: 275-284.
Borchardt MA, Stemper ME, Standridge JH. (2003). Aeromonas isolates from human diarrheic stool and groundwater compared by pulsed-field gel electrophoresis. Emerg Infect Dis 9: 224-228.

Broza M, Gancz H, Halpern M, Kashi Y. (2005). Adult non-biting midges: possible windborne carriers of Vibrio cholerae non-O1 non-O139. Environ Microbiol 7: 576-585.

Broza M, Halpern M. (2001). Chironomids egg masses and Vibrio cholerae. Nature 412: 40.

Burke V, Robinson J, Gracey M, Peterson D, Partridge K. (1984). Isolation of Aeromonas hydrophila from a metropolitan water supply: seasonal correlation with clinical isolates. Appl Environ Microbiol 48: 361-366.

Chauret C, Volk C, Creason R, Jarosh J, Robinson J, Warnes C. (2001). Detection of Aeromonas hydrophila in a drinking-water distribution system: a field and pilot study. Can J Microbiol 47: 782-786.

Felske A, Rheims H, Wolterink A, Stackebrandt E, Akkermans AD. (1997). Ribosome analysis reveals prominent activity of an uncultured member of the class Actinobacteria in grassland soils. Microbiol 143: 2983-2989.

Gavriel AA, Landre JP, Lamb AJ. (1998). Incidence of mesophilic Aeromonas within a public drinking water supply in north-east Scotland. J Appl Microbiol 84: 383-392.

Halpern M, Broza YB, Mittler S, Arakawa E, Broza M. (2004). Chironomid egg masses as a natural reservoir of Vibrio cholerae non-O1 and non-O139 in freshwater habitats. Microb Ecol 47: 341-349.

Halpern M, Gancz H, Broza M, Kashi Y. (2003). Vibriocholerae hemagglutinin/protease degrades chironomid egg masses. Appl Environ Microbiol 69: 4200-4204.

Halpern M, Gasith A, Broza M. (2002). Does the tube of a benthic chironomid larva play a role in protecting its dweller against chemical toxicants? Hydrobiologia 470: $49-55$.

Halpern M, Gasith A, Porat R, Telsch B, Broza M. (1999). Synergistic effect of chloramine and copper sulfate as control agents of planktonic midge larvae (Diptera: Chironomidae) in drinking water supply systems. J Am Mosq Control Assoc 15: 453-457.

Halpern M, Landsberg O, Raats D, Rosenberg E. (2007). Culturable and VBNC Vibrio cholerae: interactions with chironomid egg masses and their bacterial population. Microb Ecol 53: 285-293.

Halpern M, Raats D, Lavion R, Mittler S. (2006). Dependent population dynamics between chironomids (non-biting midges) and Vibrio cholerae. FEMS Microb Ecol 55: 98-104.

Handfield M, Simard P, Couillard M, Letarte R. (1996). Aeromonas hydrophila isolated from food and drinking water: hemagglutination, hemolysis, and cytotoxicity for a human intestinal cell line (HT-29). Appl Environ Microbiol 62: 3459-3461.

Holmes P, Niccolls NH, Sartory DP. (1996). The ecology of mesophilic Aeromonas in the aquatic environment. In: Austin B, Altwegg M, Gosling PJ, Joseph S (eds). The Genus Aeromonas. John Wiley \& Sons: Chichester. pp 127-150.

Huys G, Kesters I, Coopman R, Janssen P, Kersters K. (1996). Genotypic diversity among Aeromonas isolates recovered from drinking water production plants as revealed by AFLP analysis. Syst Appl Microbiol 19: 428-435. 
Kingombe CI, Huys G, Tonolla M, Albert MJ, Swings J, Peduzzi R et al. (1999). PCR detection, characterization, and distribution of virulence genes in Aeromonas spp. Appl Environ Microbiol 65: 5293-5302.

Kong RYC, Pelling A, So CL, Wu RSS. (1999). Identification of oligonucleotide primers targeted at the 16S-23S rDNA intergenic spacers for genus- and speciesspecific detection of aeromonads. Mar Pollut Bull 38: 802-888.

Kühn I, Allestam G, Huys G, Janssen P, Kersters K, Krovacek K et al. (1997a). Diversity, persistence, and virulence of Aeromonas strains isolated from drinking water distribution systems in Sweden. Appl Environ Microbiol 63: 2708-2715.

Kühn I, Huys G, Coopman R, Kersters K, Janssen P. (1997b). A 4-year study of the diversity and persistence of coliforms and Aeromonas in the water of a Swedish drinking water well. Can $J$ Microbiol 43: 9-16.

LeChevallier MW, Evans TM, Seidler RJ, Daily OP, Merrell BR, Rollins DM et al. (1982). Aeromonas sobria in chlorinated drinking water supplies. Microb Ecol 8: 325-333.

Legnani P, Leoni E, Soppelsa F, Burigo R. (1998). The occurrence of Aeromonas species in drinking water supplies of an area of the Dolomite Mountains, Italy. J Appl Microbiol 85: 271-276.
Nakano H, Kameyama T, Venkateswaran K, Kawamaki H, Hashimoto H. (1990). Distribution and characterization of hemolytic and enteropathogenic motile Aeromonas in aquatic environment. Microbiol Immunol 34: 447-458.

Nandi B, Nandy RK, Mukhopadhyay S, Nair GB, Shimada T, Ghose AC. (2000). Rapid method for species-specific identification of Vibrio cholerae using primers targeted to the gene of outer membrane protein OmpW. J Clin Microbiol 38: 4145-4151.

Schubert RH. (2000). Intestinal cell adhesion and maximum growth temperature of psychrotrophic aeromonads from surface waters. Int $J$ Hyg Environ Health 203: 83-85.

Sen K, Rodgers M. (2004). Distribution of six virulence factors in Aeromonas species isolated from US drinking water utilities: a PCR identification. J Appl Microbiol 97: 1077-1086.

Sun X-B, Cui F-Y, Zhang J-S, Xu F, Liu L-J. (2007). Inactivation of chironomid larvae with chlorine dioxide. J Hazard Mater 142: 348-353.

Tanaka N, Kobayashi T, Tanaka H, Sasa M, Kayahara I, Yamaguchi Y et al. (2004). Unexpected contamination of chironomid larvae into tap water. Pest Control Res 19: $1-5$.

Williams DA. (1974). An infestation by a parthenogenic chironomid. Wat Treat Exam 23: 215-229.

Supplementary Information accompanies the paper on The ISME Journal website (http://www.nature.com/ismej) 